Published in final edited form as:

Curr Opin Gastroenterol. 2015 May ; 31(3): 215-223. doi:10.1097/MOG.0000000000000168.

\title{
Renal dysfunction in cirrhosis
}

\author{
Nathalie H. Urrunaga ${ }^{a}$, Ayse L. Mindikoglua ${ }^{a}$, and Don C. Rockey ${ }^{b}$ \\ aDepartment of Medicine, Division of Gastroenterology and Hepatology, University of Maryland \\ School of Medicine, Baltimore, Maryland ${ }^{b}$ Department of Internal Medicine, Medical University of \\ South Carolina, Charleston, South Carolina, USA
}

\begin{abstract}
Purpose of review-Renal dysfunction causes significant morbidity in cirrhotic patients. Diagnosis is challenging because it is based on serum creatinine, which is used to calculate estimated glomerular filtration rate, which itself is not an ideal measure of renal function in patients with cirrhosis. Finding the exact cause of renal injury in patients with cirrhosis remains problematic due to the limitations of the current diagnostic tests. The purpose of this review is to highlight studies used to diagnose renal dysfunction in patients with renal dysfunction and review current treatments.
\end{abstract}

Recent findings-New diagnostic criteria and classification of renal dysfunction, especially for acute kidney injury (AKI), have been proposed in hopes of optimizing treatment and improving outcomes. New biomarkers that help to differentiate structural from functional AKI in cirrhotic patients have been developed, but require further investigation. Vasoconstrictors are the most commonly recommended treatment of hepatorenal syndrome (HRS). Given the high mortality in patients with type $1 \mathrm{HRS}$, all patients with HRS should be evaluated for liver transplantation. When renal dysfunction is considered irreversible, combined liver-kidney transplantation is advised.

Summary-Development of new biomarkers to differentiate the different types of AKI in cirrhosis holds promise. Early intervention in cirrhotic patients with renal dysfunction offers the best hope of improving outcomes.

\section{Keywords}

acute kidney injury; chronic kidney injury; hepatorenal syndrome

Copyright (C) 2015 Wolters Kluwer Health, Inc. All rights reserved.

Correspondence to Don C. Rockey, MD, Department of Internal Medicine, Medical University of South Carolina, 96 Jonathan Lucas Street, Suite 803, Charleston, SC 29425, USA. Tel: +1 843792 2914; fax: +1 843792 5265; rockey@ musc.edu.

Conflicts of interest

The authors certify that we have no financial arrangements (e.g. consultancies, stock ownership, equity interests, patent-licensing arrangements, research support, honoraria, etc.) with a company whose product figures prominently in this manuscript or with a company making a competing product. 


\section{INTRODUCTION}

Renal dysfunction is a frequent complication in cirrhotic patients, occurring in one of every five inpatients with cirrhosis [1]. Renal dysfunction in this population may present acutely, or may be a result of underlying chronic kidney disease (CKD). In any situation, it is associated with increased morbidity and mortality [2].

There are different proposed classifications of renal dysfunction in cirrhotic patients [2-5]. In general, renal dysfunction in the cirrhotic patients can be divided by acuity of presentation (acute kidney disease, acute on CKD, and CKD) and by the cause of renal dysfunction [prerenal azotemia (PRA), intrinsic renal disease, hepatorenal syndrome (HRS) type 1 and 2 and postrenal disease] (given below).

Classification of renal dysfunction in cirrhotic patients can be based on the acuity of presentation or the cause of renal dysfunction as follows:

(1) On the basis of acuity of presentation

(a) Acute kidney injury

(b) Chronic kidney disease

(c) Acute on chronic kidney disease

(2) On the basis of the cause of renal dysfunction

(a) Prerenal azotemia

(b) HRS type 1

(c) Intrinsic renal disease

(i) Acute tubular necrosis (ATN), glomerulopathies, acute interstitial nephritis (AIN), contrast-induced nephropathy (the list is not exhaustive)

(d) Postrenal disease.

Most patients with cirrhosis and renal dysfunction have an acute presentation without renal structural changes. For example, a study of 152 inpatients with cirrhosis found that $70 \%$ of the patients had acute kidney injury (AKI), 17\% AKI and CKD, and 13\% had CKD alone [6]. Unfortunately, patients who do not improve with initial treatment often progress to HRS and have increased mortality [7].

The diagnosis of AKI and CKD is challenging as it relies on serum creatinine used to calculate estimated glomerular filtration rate (eGFR), and several investigations have shown that serum creatinine is not an accurate biomarker of renal function in patients with cirrhosis [8]. Most importantly, finding the exact cause of renal injury in patients with cirrhosis is still a clinical dilemma due to the limitations of the current available diagnostic tests.

The review will highlight recent data on classification, pathophysiology, testing, and treatment in patients with cirrhosis and renal dysfunction, with a focus on AKI. 


\section{DEFINITIONS OF RENAL DYSFUNCTION IN CIRRHOSIS}

The Acute Dialysis Quality Initiative (ADQI) group and International Ascites Club (IAC) formed a Working Group and published a classification in 2011 that proposed the following classification of renal dysfunction in patients with cirrhosis: AKI is defined as a rise in serum creatinine of more than $50 \%$ from baseline or an increase in creatinine of at least 0.3 $\mathrm{mg} / \mathrm{day}$ in less than $48 \mathrm{~h}$, (this definition is independent of the cause of acute renal dysfunction) [2]; CKD is defined as eGFR of less than $60 \mathrm{ml} / \mathrm{min}$ for more than 3 months; and acute on CKD is defined as the increase in serum creatinine of at least $50 \%$ from baseline or the increase in creatinine by at least $0.3 \mathrm{mg} / \mathrm{dl}$ in less than $48 \mathrm{~h}$ in a cirrhotic patient with GFR below $60 \mathrm{ml} / \mathrm{min}$ for more than 3 months [2].

Causes of AKI include prerenal, intrinsic renal or parenchymal disorders, obstructive nephropathy, and HRS [1]. The classic diagnostic criteria for HRS includes the presence of cirrhosis, typically complicated by ascites, serum creatinine above $1.5 \mathrm{mg} / \mathrm{dl}$ (or $>133$ $\mu \mathrm{mol} / \mathrm{l})$, discontinuation of diuretics for 2 days, correction of volume depletion, no improvement of creatinine after 2 days of albumin $(1 \mathrm{~g} / \mathrm{kg}$ body weight/day, up to a maximum of $100 \mathrm{~g} / \mathrm{day}$ ), the absence of nephrotoxic medications, and the absence of findings suggestive of parenchymal renal disease (which include proteinuria or urinary excretion of $>500 \mathrm{mg}$ of protein/day, $>50$ red cells/high-power field, or abnormal renal ultrasound) [7,9]. There are two types of HRS - type 1 is defined as a doubling of the baseline serum creatinine to greater than $2.5 \mathrm{mg} / \mathrm{dl}(221 \mu \mathrm{mol} / \mathrm{l})$ or a $50 \%$ decrease in the initial 24-h creatinine clearance to less than $20 \mathrm{ml} / \mathrm{min}$ in less than 2 weeks, whereas type 2 is characterized by a relatively stable renal dysfunction and/or less rapidly progressive renal dysfunction [7]. In typical type 1 HRS, the structure of the kidney is normal. It should be pointed out that a clear definition of type 2 HRS is problematic since it is poorly characterized clinically [6].

PRA is generally caused by decreased perfusion to the kidney. PRA is the most common cause of acute renal failure in patients with cirrhosis [6,10]. Multiple factors can contribute to PRA, including gastrointestinal bleeding, overdiuresis, excessive diarrhea due to lactulose use or infectious causes, and hypotension secondary to beta blocker use or ACE inhibitors. PRA is usually related to urinary specific gravity less than 1.020 and fractional excretion of sodium (FENa) less than 1\% [6]. Other supporting clinical findings include tachycardia, hypotension, and history of exacerbating factors, as mentioned above.

Causes of intrinsic or structural renal disease in patients with cirrhosis include primarily ATN and the glomerulopathies. Glomerular diseases seen in cirrhotic patients include membranous nephropathy and membranoproliferative glomerulonephritis, both seen in patients with hepatitis B and C; IgA nephropathy reported in alcoholic patients; and diabetic glomerulosclerosis. The exact frequency of each cause in patients with cirrhosis has not been described yet. Other causes of structural renal disease include AIN and ischemic nephropathy $[3,10]$.

ATN in cirrhotic patients is usually related to volume depletion (i.e. relative hypotension) caused by gastrointestinal bleeding, overdiuresis, or large-volume paracentesis [6]. Also, 
antibiotics, contrast nephropathy, and prolonged ischemia secondary to HRS can lead to ATN [11]. The differential diagnosis between HRS and ATN remains challenging, but classically, the presence of renal tubular epithelial cells in the urine sediment and FENa greater than $2 \%$ are associated with the diagnosis of ATN [1].

\section{PATHOPHYSIOLOGY OF RENAL DYSFUNCTION IN CIRRHOSIS}

In early cirrhosis, when portal hypertension develops, a number of changes occur, and these have been well described previously. In brief, altered vascular biology in the liver and periphery triggers a series of changes including arterial vasodilation in the splanchnic circulation, which causes peripheral systemic vascular vasoconstriction [12"]. Splanchnic vasodilation causes secondary peripheral vasoconstriction, and activation of the reninangiotensin-aldosterone system (RAAS) and the sympathetic nervous system (SNS), which increases secretion of vasopressin in an attempt to keep arterial blood pressure with subsequent peripheral vasoconstriction [13]. Additionally, splanchnic vasodilation exacerbates a phenomenon of increased hyper-dynamic circulation which promotes cardiovascular dysfunction followed by decrease in the cardiac output and worsening peripheral vasoconstriction. This contributes to changes in the heart including myocardial hypertrophy and diastolic dysfunction, which is also known as cirrhotic cardiomyopathy [14].

When the GFR decreases, there is a further dysregulation of the RAAS, with increased levels of aldosterone and renin; this leads to sodium retention and worsening of ascites and fluid overload [5]. Vasopressin is also stimulated locally and centrally, further leading to a series of changes that result in water retention by the nephron [15]. There may also be increased renal vasoconstriction and a reduction in renal perfusion with subsequent renal ischemia, and in turn, reduced GFR, which predisposes to the development of HRS [16].

Another important factor in the pathogenesis of AKI in patients with cirrhosis is the systemic inflammatory response, with or without infection. Patients with cirrhosis and ascites have a high risk for bacterial translocation from the intestinal lumen to the abdominal lymph nodes, which leads to increased circulating levels of lipopolysaccharide-binding protein (LBP) and tumor necrosis factor-alpha (TNF-a) (which in turn may cause splanchnic vasodilation). After liver injury, injured hepatocytes release damage-associated molecular pattern (DAMP) molecules, which appear to exacerbate inflammatory response. High mobility group box-1 (HMGB1) is a nonhistone nuclear protein that functions as a DAMP molecule and is observed in high levels after liver ischemia and reperfusion [17]. HMGB1 appears to interact with toll-like receptors (TLRs), including TLR-2 and TLR-4, thus leading to renal endothelial cell injury and causing renal injury, most specifically in the proximal tubule [17]. Additionally, it has been found that after liver ischemia, intestinal Paneth cells secrete large amounts of interleukin (IL)-17A which can be found in the circulation and which appears to be deposited in the kidney leading to kidney injury [18].

Several exogenous factors can trigger acute renal dysfunction, including drugs such as NSAIDs, angiotensin-converting-enzyme inhibitors (ACEIs), and diuretics. Other factors 
such as large-volume paracentesis, any type of infection, and gastrointestinal hemorrhage have been associated with acute renal dysfunction in this population [19].

A recent study that included patients with decompensated cirrhosis showed that the risk factors for AKI are similar to those for HRS [20]. Precipitating factors included largevolume paracentesis ( $>51$ ), increased diuretic doses, spontaneous bacterial peritonitis, other bacterial infections, intravenous contrast, variceal bleed, and nonvariceal gastrointestinal bleed [20]. The findings were suggestive that AKI and HRS in cirrhotic patients may share similar pathophysiological pathways [20]. Importantly, once AKI develops, even small increases in GFR are associated with reduction in patient survival. Some patients recover renal function after AKI, but some progress to HRS [20].

\section{DIAGNOSIS}

In order to accurately assess renal function, it is important to calculate GFR. Serum creatinine is the most commonly used marker to calculate GFR and estimate renal function, but unfortunately it overestimates GFR in decompensated cirrhotic patients. For example, patients with cirrhosis often have reduced muscle mass, reduced synthesis of creatine (the precursor of creatinine), and additionally, elevated bilirubin levels may interfere with creatinine measurements [21,22]. GFR calculation by clearance of inulin or radioisotopic markers like ${ }^{125}$ Iothalamate or ${ }^{99} \mathrm{Tc}$-diethylene triamine pentacetic acid (DTPA) appears to be the most accurate method to assess GFR; however, these tests are time-consuming and are not practical in daily clinical practice [23"']. Importantly, the use of serum creatinine may delay early diagnosis of renal dysfunction and thus may delay initiation of appropriate treatment for acute renal injury and HRS type $1[2,24,25]$.

In an attempt to minimize delay in diagnosis, the ADQI-IAC formed a Working Group and proposed a new term called 'hepatorenal disorders' (HRDs), which included all types of renal dysfunction in patients with decompensated cirrhosis. These included functional and structural renal dysfunction in patients with AKI, CKD, or HRS. In this definition, type 1 HRS is considered a type of AKI, but these new diagnostic criteria of AKI in patients with cirrhosis need to be validated; at present, they are considered to be based only on expert opinion [2] and the use of the current definition of HRS is still recommended. Future studies will need to confirm the benefit of early diagnosis, in which case it is likely that the creatinine cut-off for the diagnosis of HRS (especially type 1) may need to be revised.

Since creatinine measurement in cirrhotic patients remains imperfect, a number of investigators have examined newer methodologies that might better estimate GFR. For example, several recent studies have assessed the performance of serum creatinine-based and cystatin C-based equations, and compared these with measured GFR. In one study of 300 patients who were evaluated for liver transplantation, GFR was directly measured by using plasma clearance of iohexol. Modified Diet in Renal Disease (MDRD)-4, MDRD-6, and Chronic Kidney Disease Epidemiology Collaboration (CKD-EPI) equations to calculate eGFR were used. MDRD-4 and CKD-EPI overestimated GFR, whereas MDRD-6 appeared to be the most accurate equation to estimate GFR [26"']. 
Formulas using cystatin C to estimate GFR take advantage of the biology of cystatin C. This cysteine proteinase inhibitor is produced at a constant rate by all nucleated cells [27] and is filtrated completely by the glomeruli and can be measured in serum and urine. Despite the fact that, its expression may be influenced by nonrenal factors such as sepsis, inflammation, steroids, smoking, thyroid disease, age, and sex, cystatin $\mathrm{C}$ is considered a better marker of glomerular filtration than creatinine [28]. Recent studies have shown that serum cystatin C equations and cystatin $\mathrm{C}-$ creatinine equations performed better than regular creatinine equations in calculating GFR [23"',29"']. A study that included 202 patients with cirrhosis in whom inulin clearance was used to calculate GFR compared the performance of creatinine, cystatin C, and creatinine-cystatin C equations with the measured GFR (including MDRD-4, MDRD-6, CKD-EPI-cystatin C, CKD-EPI- creatinine-cystatin C). Cystatin C equations, especially CKD-EPI cystatin $\mathrm{C}$ equation, performed best irrespective of cirrhosis severity [29"'].

Another study examined 72 patients with cirrhosis in whom GFR was measured by nonradiolabeled iothalamate plasma clearance (mGFR). Comparison of creatinine, cystatin $\mathrm{C}$-based, and the CKD-EPI creatinine-cystatin $\mathrm{C}$ equations revealed that the new CKD-EPI creatinine-cystatin $\mathrm{C}$ equation was superior to other equations for estimating GFR in patients with cirrhosis [23"']. Despite these encouraging findings, more studies are needed to test the utility and accuracy of these equations in patients with decompensated cirrhosis.

The differential diagnosis of a patient with cirrhosis and AKI includes primarily PRA, ATN, and HRS [30"']. While making a specific diagnosis and identification of the cause of AKI is important to appropriately direct treatment, currently available diagnostic tests limit the clinician's accuracy. A rapidly expanding body of literature indicates that urine markers of injury may improve the ability to diagnose renal injury in patients with cirrhosis (listed below).

New kidney biomarkers (measured in urine) under investigation for the diagnosis of AKI in patients with cirrhosis include the following tubular damage biomarkers:

(1) Neutrophil gelatinase-associated lipocalin (NGAL)

(2) Kidney injury molecule-1 (KIM-1)

(3) IL-18

(4) Liver type fatty acid-binding protein (L-FABP).

The new urinary biomarkers proposed include NGAL,IL-18,KIM-1, andLFABP, whichareassociated with tubular injury; and albumin, which is associated with glomerular injury - both groups indicate structural kidney damage suggestive of ATN [31].

A recent study evaluated biomarkers in 110 patients with cirrhosis and AKI [30"']. Patients were diagnosed with PRA, ATN, and HRS by a group of experts after retrospective analysis of the clinical course and laboratory data which excluded measurements of NGAL, IL-18, KIM-1, L-FABP, and albumin. ATN was found in 53\%, PRA in 26\%, and HRS in $22 \%$ of the patients. NGAL, IL-18, KIM-1, L-FABP, and albumin were significantly higher in patients with ATN than in patients with PRA or HRS. The likelihood of a diagnosis of ATN 
increased with the number of biomarkers above optimal diagnostic cut-offs. This suggests that these new biomarkers can be helpful to exclude patients with significant structural renal injury who are unlikely to benefit from treatment for HRS or PRA [30"']. Another study that included 118 patients with cirrhosis and measured urinary NGAL found that $44 \%$ of the patients had normal kidney function, $12 \%$ had stable CKD, 14\% PRA, 17\% HRS, and 13\% intrinsic AKI (defined as acute elevation in creatinine to $>1.5$ or $>0.3 \mathrm{mg} / \mathrm{dl}$ above baseline and no response to $48 \mathrm{~h}$ of volume resuscitation and, not meeting diagnostic criteria for HRS). The study found that patients with normal renal function had a median urinary neutrophil gelatinase-associated lipocalin (uNGAL) level of $20 \mathrm{ng} / \mathrm{ml}$, patients with PRA had a median uNGAL level of $20 \mathrm{ng} / \mathrm{ml}$, patients with CKD had a median uNGAL level of $50 \mathrm{ng} / \mathrm{ml}$, patients with HRS had a median uNGAL level of $105 \mathrm{ng} / \mathrm{ml}$, and patients with intrinsic AKI had a median uNGAL level of $325 \mathrm{ng} / \mathrm{ml}$ [32]. Unfortunately, these biomarkers are currently used for investigational purposes only.

Also, noninvasive biomarkers of renal plasma flow (RPF) and renal resistive indices (measured by duplex Doppler ultrasonography) have been explored as possible predictors of HRS in patients with cirrhosis. Reduction in renal cortical blood circulation is one of the main changes that precede HRS and decrease in GFR. One study showed reduced RPF, reduced filtration fraction (or GFR/RPF), and increased renal resistive indices (right kidney main and arcuate artery resistive indices) in patients with cirrhosis and ascites at higher risk for HRS [33]. Given the small size of the study, further investigation is needed to evaluate the utility of these biomarkers in patients with cirrhosis.

The diagnostic evaluation of AKI in patients with cirrhosis includes the following: a complete history (with review of medication use), physical examination, exclusion of postrenal obstruction, correction of PRA, if present, and careful monitoring of serum creatinine. In most patients, the urine should be examined with microscopy for urinespecific gravity, granular or epithelial casts, leukocytes, nitrites, leukocyte esterase, electrolytes, and urine protein measured (spot and if abnormal 24-h urine collection is recommended). In any patient with cirrhosis and AKI, infectious processes also should be considered as a cause of PRA. Complete blood count, diagnostic paracentesis in patients with ascites (including ascitic fluid cell count and culture), blood culture, urine culture, and chest radiograph should be considered to exclude infection sources [5]. In many patients, these studies will point to potential intrinsic renal disease, which would then be explored with other specific diagnostic testing.

\section{TREATMENT}

Early treatment of renal dysfunction in cirrhotic patients, especially in patients with acute deterioration, is important and may improve outcomes. Early treatment may even be initiated before a cause of renal dysfunction is firmly established. NSAIDS and ACEIs should be avoided in cirrhotic patients and stopped if the patients were taking them. Also, loop diuretics and potassium-sparing diuretics should be discontinued until renal function recovers. If patients have severe ascites, large-volume paracentesis with albumin infusion at $8 \mathrm{~g} / \mathrm{l}$ of ascitic fluid removed can be considered as this may remove pressure on the inferior 
vena cava and improve blood return to the central circulation [34]. Once a cause of renal dysfunction is determined, more specific treatment(s) can be implemented (Fig. 1).

\section{Prerenal azotemia}

Intravascular depletion should be treated with fluid resuscitation and/or albumin infusion. In patients with gastrointestinal bleeding, transfusion of blood products should be considered, with a hemoglobin goal of $7 \mathrm{~g} / \mathrm{dl}$ [35]. Medications exacerbating PRA should be stopped.

\section{Hepatorenal syndrome}

Correction of triggering factors, including infectious processes or hypovolemia and vasoconstrictors, is essential. A fluid challenge with intravenous (i.v.) albumin at $1 \mathrm{~g} / \mathrm{kg} / \mathrm{day}$ (or maximum $100 \mathrm{~g}$ /day divided into 3-4 doses/day) for 2 days should be started. If renal dysfunction does not improve, vasoconstrictors should be considered. Vasoconstrictors for treatment of HRS such as terlipressin can restore renal function in almost 40-50\% of the cirrhotic patients [5]. Some authors have proposed that treatment with vasoconstrictors should be started if AKI does not improve with volume resuscitation, even before they meet criteria for HRS $[19,20]$. Vasoconstrictor agents such as vasopressin analogs and a1 agonists are the most commonly recommended treatment option for HRS.

Terlipressin is a synthetic vasopressin analog that has long been used in patients with HRS (primarily type 1). Although most published studies excluded patients with a high risk of ischemia, ischemic events have been reported in up to $30 \%$ of the patients. Terlipressin is started at $0.5-1 \mathrm{mg}$ every $4-6 \mathrm{~h}$ i.v., and it is increased up to $2 \mathrm{mg}$ every $4-6 \mathrm{~h}$, if there is no improvement of serum creatinine of at least $25 \%$ by day 3 of treatment. Treatment is continued until the creatinine level decreases below $1.5 \mathrm{mg} / \mathrm{dl}(133 \mu \mathrm{mol} / \mathrm{l})$ to a maximum of 15 days. In majority of the studies, albumin was administered (at $1 \mathrm{~g} / \mathrm{kg}$ on day 1 followed by $40 \mathrm{~g} /$ day) along with terlipressin [36]. Terlipressin is not available in the United States.

A recent meta-analysis that included a total of five randomized clinical studies evaluated terlipressin for type 1 HRS [37]. Mortality was $48 \%$ in patients who received terlipressin alone or terlipressin with albumin, vs. $64 \%$ in patients randomized to no intervention, placebo, or albumin alone; it thus reduced mortality with a relative risk (RR) of 0.76 [95\% confidence interval (CI) 0.61-0.95]. In general, the assessment of mortality was limited due to relatively small numbers of patients included and the short follow-up periods (two studies followed up to the end of the treatment, one for 3 months after the end of the treatment and two for 6 months after the end of the treatment). Among the assessed adverse events, only cardiovascular adverse events were higher in patients who received terlipressin [37].

A recent prospective study that included 18 patients with type 1 HRS and sepsis who received terlipressin and albumin showed that there was a significant improvement in arterial blood pressure and suppression of high renin levels and norepinephrine [38"]. Improvement of renal function was observed in $67 \%$ of the patients and was associated with an improved 3-month survival compared to patients without response. Patients who did not respond had a significantly lower baseline heart rate, higher Model for End-Stage Liver Disease (MELD) and Child-Pugh scores, and higher values of chronic liver failuresequential organ failure assessment (CLIF-SOFA) score, which indicated greater severity of 
the acute-on-chronic liver failure (ACLF). A CLIF-SOFA score of at least 11 had a 100\% specificity and $92 \%$ sensitivity in predicting no response to treatment [38"].

In the United States, since terlipressin is not available, other vasoconstrictor agents have been used. For example, the a 1 adrenergic receptor agonist midodrine (combined with octreotide and albumin infusion) has been examined in small studies. One study of five patients with cirrhosis and HRS who received 10-20 g of albumin per day for 20 days, and octreotide (started at $100 \mathrm{mg}$ three times per day and titrated up to a target dose of $200 \mu \mathrm{g}$ subcutaneously three times per day) and midodrine (started at $7.5 \mathrm{mg}$ three times per day and titrated up to a maximum of $12.5 \mathrm{mg}$ orally three times per day) led to reversal of HRS in all patients [39]. Another study using i.v. octreotide infusion at $25 \mathrm{mg} / \mathrm{h}$ and a fixed dose of midodrine orally ( $2.5 \mathrm{mg} /$ day) in 14 patients with type $1 \mathrm{HRS}$ revealed that serum creatinine decreased to less than $1.5 \mathrm{mg} / \mathrm{dl}$ in $70 \%$ of the patients [40]. A retrospective study that included 60 patients treated with the combination octreotide, midodrine and albumin, vs. 21 control patients treated with albumin showed reduction in mortality in the triple combination group (43 vs.71\%; $P<0.05$ ) [41].

Norepinephrine is also used for the treatment of HRS [42]. Norepinephrine is used as a continuous infusion (starting at $0.5 \mathrm{mg} / \mathrm{h}$ and titrated up to obtain $10 \mathrm{mmHg}$ increase in the mean arterial blood pressure or increase in urinary output $>200 \mathrm{ml} / 4 \mathrm{~h}$ ). In one study, it was used with albumin and furosemide in 12 patients with type 1 HRS until creatinine decreased to less than $1.5 \mathrm{mg} / \mathrm{dl}$. Eighty-three percent of patients responded to treatment. Ischemic complications were reported in $17 \%$ of the patients [42].

A recent systematic review examined the major vasoconstrictors available for HRS, focusing on terlipressin and norepinephrine. In this review of four studies and a total of 154 patients, it was found that terlipressin and norepinephrine appeared to be equivalent in terms of HRS reversal, mortality at 30 days, and recurrence of HRS. Of note, adverse events were less frequent in patients who received norepinephrine [43].

Renal replacement therapy (RRT) is recommended in patients who are waiting for a liver transplant and develop severe metabolic acidosis, volume overload, or hyperkalemia. Side effects include hypotension, bleeding, and infections [5]. Whether RRT decreases mortality is still unclear, although limited data suggest that mortality is lower in patients with HRS and receiving RRT. For example, one retrospective study that included 26 patients with HRS showed that $44 \%$ of patients on RRT survived to liver transplantation vs. only $10 \%$ in the group who did not receive RRT [44].

The molecular adsorbent recirculating system (MARS) is a form of RRT that combines continuous RRT (CRRT) and an albumin-enriched dialysate. It has been hypothesized that MARS can remove toxins bound to albumin, including nitric oxide and bile acids and cytokines like IL-6 and TNF-a. A small study has shown evidence of improvement in survival at 7 and 30 days when compared to conventional therapy [45]. Another study that included five patients with cirrhosis and type 1 HRS who failed vasoconstrictor therapy showed that despite a significant decrease in nitric oxide levels, MARS did not improve systemic hemodynamics or GFR [46]. Transjugular intrahepatic portosystemic shunt (TIPS) 
has been associated with improvement in the renal function in select patients with HRS [40,47,48], but it should be emphasized that this is risky in patients with severe liver dysfunction and is not generally considered a standard practice. Larger randomized controlled clinical trials are needed to evaluate MARS and TIPS for the treatment of HRS.

A retrospective study that included 62 patients with type 1 HRS showed that postliver transplant HRS resolved in $76 \%$ of the patients in a mean time of 13 days. The only predictor of HRS nonreversal was duration of dialysis in the pretransplant period, with a $6 \%$ increase in risk of nonreversal with each additional day of dialysis [49"]. Given the high mortality in patients with HRS, especially type $1 \mathrm{HRS}$, it is recommended that all patients with HRS type 1 and 2 should be evaluated for liver transplantation if they have no major contraindications. If renal failure is considered irreversible, combined liver-kidney transplantation is advised. The current United Network for Organ Sharing recommendations for combined liver-kidney transplantation include: CKD requiring dialysis, CKD not requiring dialysis and evidence of proteinuria, sustained AKI on RRT for 6 weeks or more (at least twice a week), sustained AKI (with GFR $\leq 25 \mathrm{ml} / \mathrm{min}$ ) not on RRT for 6 weeks or more, and metabolic disease [50].

\section{CONCLUSION}

Several recent studies have provided new information about the diagnosis and management of patients with cirrhosis and renal dysfunction. Cystatin $\mathrm{C}$ is a cysteine proteinase inhibitor that is produced by a constant secretion rate by all nucleated cells and is reliably filtered by glomeruli; serum cystatin C-based equations, and cystatin C-creatinine equations may prove to better estimate GFR than serum creatinine-based equations in patients with cirrhosis.

NGAL and other new biomarkers have been the potential to differentiate HRS from structural kidney disease, although their use is currently limited to investigational purposes. The management of AKI and HRS follows a specific algorithm, including ensuring that patients are adequately volume-repleted, treated for infection when it is present, and that all potential nephrotoxins have been removed. The use of vasoconstrictor agents is the most commonly recommended treatment option for HRS. All patients with type 1 HRS should be considered for liver transplantation. Further research is expected to shed further light on the role of early diagnosis and treatment and outcomes. Additionally, it is expected that new biomarkers will also help facilitate and expedite diagnosis and aggressive treatment.

\section{Acknowledgements}

None.

Financial support and sponsorship The study was supported in part by a T32 NIH Research Grant DK 067872 (Clinical Research Fellowship to N.H.U.) and by the Grant Number 5 K23 DK089008-05 from the National Institutes of Health (NIH), National Institute of Diabetes and Digestive and Kidney Diseases (to A.L.M.) and its contents are solely the responsibility of the authors and do not necessarily represent the official views of the National Institute of Diabetes and Digestive and Kidney Diseases or the NIH.

\section{REFERENCES AND RECOMMENDED READING}

Papers of particular interest, published within the annual period of review, have been highlighted as: 
- of special interest

- of outstanding interest

1. Garcia-Tsao G, Parikh CR, Viola A. Acute kidney injury in cirrhosis. Hepatology. 2008; 48:20642077. [PubMed: 19003880]

2. Wong F, Nadim MK, Kellum JA, et al. Working Party proposal for a revised classification system of renal dysfunction in patients with cirrhosis. Gut. 2011; 60:702-709. [PubMed: 21325171]

3. Francoz C, Glotz D, Moreau R, Durand F. The evaluation of renal function and disease in patients with cirrhosis. J Hepatol. 2010; 52:605-613. [PubMed: 20185192]

4. Mindikoglu AL, Weir MR. Current concepts in the diagnosis and classification of renal dysfunction in cirrhosis. Am J Nephrol. 2013; 38:345-354. [PubMed: 24107793]

5. Ginès P, Schrier RW. Renal failure in cirrhosis. N Engl J Med. 2009; 361:1279-1290. [PubMed: 19776409]

6. Warner NS, Cuthbert JA, Bhore R, Rockey DC. Acute kidney injury and chronic kidney disease in hospitalized patients with cirrhosis. J Investig Med. 2011; 59:1244-1251.

7. Salerno F, Gerbes A, Ginès P, et al. Diagnosis, prevention and treatment of hepatorenal syndrome in cirrhosis. Gut. 2007; 56:1310-1318. [PubMed: 17389705]

8. Caregaro L, Menon F, Angeli P, et al. Limitations of serum creatinine level and creatinine clearance as filtration markers in cirrhosis. Arch Intern Med. 1994; 154:201-205. [PubMed: 8285815]

9. Arroyo V, Ginès P, Gerbes AL, et al. Definition and diagnostic criteria of refractory ascites and hepatorenal syndrome in cirrhosis. International Ascites Club. Hepatology. 1996; 23:164-176. [PubMed: 8550036]

10. Sampaio MS, Martin P, Bunnapradist S. Renal dysfunction in end-stage liver disease and postliver transplant. Clin Liver Dis. 2014; 18:543-560. [PubMed: 25017075]

11. Schrier RW, Shchekochikhin D, Ginès P. Renal failure in cirrhosis: prerenal azotemia, hepatorenal syndrome and acute tubular necrosis. Nephrol Dial Transplant. 2012; 27:2625-2628. [PubMed: 22492830]

12-. Iwakiri Y, Shah V, Rockey DC. Vascular pathobiology in chronic liver disease and cirrhosis current status and future directions. J Hepatol. 2014; 61:912-924. [PubMed: 24911462] A stateof-the-art review of multiple aspects of the pathophysiology of portal hypertension.

13. Epstein M, Levinson R, Sancho J, et al. Characterization of the renin-aldosterone system in decompensated cirrhosis. Circ Res. 1977; 41:818-829. [PubMed: 923033]

14. Ruiz-del-Arbol L, Monescillo A, Arocena C, et al. Circulatory function and hepatorenal syndrome in cirrhosis. Hepatology. 2005; 42:439-447. [PubMed: 15977202]

15. Ivarsen P, Frøkiaer J, Aagaard NK, et al. Increased urinary excretion of aquaporin 2 in patients with liver cirrhosis. Gut. 2003; 52:1194-1199. [PubMed: 12865281]

16. Arroyo V, Colmenero J. Ascites and hepatorenal syndrome in cirrhosis: pathophysiological basis of therapy and current management. J Hepatol. 2003; 38(Suppl 1):S69-S89. [PubMed: 12591187]

17. Lee HT, Park SW, Kim M, D’Agati VD. Acute kidney injury after hepatic ischemia and reperfusion injury in mice. Lab Invest. 2009; 89:196-208. [PubMed: 19079326]

18. Park SW, Kim M, Brown KM, et al. Paneth cell-derived interleukin-17A causes multiorgan dysfunction after hepatic ischemia and reperfusion injury. Hepatology. 2011; 53:1662-1675. [PubMed: 21360570]

19. Leithead JA, Hayes PC, Ferguson JW. Review article: advances in the management of patients with cirrhosis and portal hypertension-related renal dysfunction. Aliment Pharmacol Ther. 2014; 39:699-711. [PubMed: 24528130]

20. Tsien CD, Rabie R, Wong F. Acute kidney injury in decompensated cirrhosis. Gut. 2013; 62:131137. [PubMed: 22637695]

21. Sherman DS, Fish DN, Teitelbaum I. Assessing renal function in cirrhotic patients: problems and pitfalls. Am J Kidney Dis. 2003; 41:269-278. [PubMed: 12552488]

22. Daugherty NA, Hammond KB, Osberg IM. Bilirubin interference with the kinetic Jaffé method for serum creatinine. Clin Chem. 1978; 24:392-393. [PubMed: 627087] 
23-. Mindikoglu AL, Dowling TC, Weir MR, et al. Performance of chronic kidney disease epidemiology collaboration creatinine-cystatin $\mathrm{C}$ equation for estimating kidney function in cirrhosis. Hepatology. 2014; 59:1532-1542. [PubMed: 23744636] This cohort study of 72 patients with cirrhosis in whom GFR was measured by nonradiolabeled iothalamate plasma clearance (mGFR) and compared with other creatinine, cystatin C-based equations and the new CKD-EPI creatinine-cystatin $\mathrm{C}$ equation showed that the diagnostic performance of CKD-EPI creatinine-cystatin $\mathrm{C}$ equation in patients with cirrhosis was superior to other equations.

24. Moore K. Acute kidney injury in cirrhosis: a changing spectrum. Hepatology. 2013; 57:435-437. [PubMed: 22886711]

25. Belcher JM, Parikh CR, Garcia-Tsao G. Acute kidney injury in patients with cirrhosis: perils and promise. Clin Gastroenterol Hepatol. 2013; 11:1550-1558. [PubMed: 23583467]

26-m. Francoz C, Nadim MK, Baron A, et al. Glomerular filtration rate equations for liver-kidney transplantation in patients with cirrhosis: validation of current recommendations. Hepatology. 2014; 59:1514-1521. [PubMed: 24037821] This study included 300 patients with cirrhosis who were evaluated for liver transplantation. GFR was measured by using plasma clearance of iohexol and compared with eGFR calculated with the MDRD-4, MDRD-6, and CKD-EPI equations. MDRD-4 and CKD-EPI overestimated GFR, whereas MDRD-6 was the most accurate equation to estimate GFR.

27. Filler G, Bökenkamp A, Hofmann W, et al. Cystatin C as a marker of GFR: history, indications, and future research. Clin Biochem. 2005; 38:1-8. [PubMed: 15607309]

28. Parikh CR, Belcher JM. Reconsidering a 'chopped liver': the need for improving glomular filtration rate estimation for hepatic transplantation. Hepatology. 2014; 59:1242-1245. [PubMed: 24470267]

29m. De Souza V, Hadj-Aissa A, Dolomanova O, et al. Creatinine- versus cystatine C-based equations in assessing the renal function of candidates for liver transplantation with cirrhosis. Hepatology. 2014; 59:1522-1531. [PubMed: 24123197] This study evaluated 202 patients with cirrhosis in whom inulin clearance was measured to calculate GFR. The performance of creatinine, cystatin $\mathrm{C}$ and creatinine-cystatin $\mathrm{C}$ equations was evaluated. Cystatin $\mathrm{C}$ equations, especially CKD-EPI cystatin $\mathrm{C}$ equation, performed the best, irrespective of cirrhosis severity.

30m. Belcher JM, Sanyal AJ, Peixoto AJ, et al. Kidney biomarkers and differential diagnosis of patients with cirrhosis and acute kidney injury. Hepatology. 2014; 60:622-632. [PubMed: 24375576] This multicenter retrospective study examined 110 patients with cirrhosis and AKI. Patients were diagnosed with PRA, ATN, and HRS by a group of experts after retrospective analysis of the clinical course and laboratory data which excluded measurements of urinary NGAL, IL-18, KIM-1, L-FABP, and albumin. NGAL, IL-18, KIM-1, L-FABP, and albumin were significantly higher in patients with ATN than in patients with PRA or HRS. The likelihood of a diagnosis of ATN increased with the number of biomarkers above optimal diagnostic cutoffs. This suggests that these new biomarkers can be helpful to exclude patients with significant structural renal injury who are unlikely to benefit from treatment for HRS or PRA.

31. Wong F, Murray P. Kidney damage biomarkers: novel tools for the diagnostic assessment of acute kidney injury in cirrhosis. Hepatology. 2014; 60:455-457. [PubMed: 24700173]

32. Verna EC, Brown RS, Farrand E, et al. Urinary neutrophil gelatinase-associated lipocalin predicts mortality and identifies acute kidney injury in cirrhosis. Dig Dis Sci. 2012; 57:2362-2370. [PubMed: 22562534]

33. Mindikoglu AL, Dowling TC, Wong-You-Cheong JJ, et al. A pilot study to evaluate renal hemodynamics in cirrhosis by simultaneous glomerular filtration rate, renal plasma flow, renal resistive indices and biomarkers measurements. Am J Nephrol. 2014; 39:543-552. [PubMed: 24943131]

34. Huggins JT, Doelken P, Walters C, Rockey DC. Point-of-care-echocardiography improves assessment of volume status in cirrhosis and hepatorenal syndrome. Am J Med Sci. (in press).

35. Rockey DC. To transfuse or not to transfuse in upper gastrointestinal hemorrhage? That is the question. Hepatology. 2014; 60:422-424. [PubMed: 24390775]

36. Liver EAftSot. EASL clinical practice guidelines on the management of ascites, spontaneous bacterial peritonitis, and hepatorenal syndrome in cirrhosis. J Hepatol. 2010; 53:397-417. [PubMed: 20633946] 
37. Gluud LL, Christensen K, Christensen E, Krag A. Terlipressin for hepatorenal syndrome. Cochrane Database Syst Rev. 2012; 9:CD005162. [PubMed: 22972083]

38-. Rodríguez E, Elia C, Solà E, et al. Terlipressin and albumin for type-1 hepatorenal syndrome associated with sepsis. J Hepatol. 2014; 60:955-961. [PubMed: 24447876] This prospective study included 18 patients with type 1 HRS and sepsis who received terlipressin and albumin, and showed that there was a significant improvement in arterial blood pressure and suppression of high renin levels and norepinephrine. Improvement of renal function was obtained in $67 \%$ of the patients and this was associated with improved 3-month survival compared to patients without response.

39. Angeli P, Volpin R, Gerunda G, et al. Reversal of type 1 hepatorenal syndrome with the administration of midodrine and octreotide. Hepatology. 1999; 29:1690-1697. [PubMed: 10347109]

40. Wong F, Pantea L, Sniderman K. Midodrine, octreotide, albumin, and TIPS in selected patients with cirrhosis and type 1 hepatorenal syndrome. Hepatology. 2004; 40:55-64. [PubMed: 15239086]

41. Esrailian E, Pantangco ER, Kyulo NL, et al. Octreotide/Midodrine therapy significantly improves renal function and 30-day survival in patients with type 1 hepatorenal syndrome. Dig Dis Sci. 2007; 52:742-748. [PubMed: 17235705]

42. Duvoux C, Zanditenas D, Hézode C, et al. Effects of noradrenalin and albumin in patients with type I hepatorenal syndrome: a pilot study. Hepatology. 2002; 36:374-380. [PubMed: 12143045]

43. Nassar Junior AP, Farias AQ, D’Albuquerque LA, et al. Terlipressin versus norepinephrine in the treatment of hepatorenal syndrome: a systematic review and meta-analysis. PLoS One. 2014; 9:e107466. [PubMed: 25203311]

44. Keller F, Heinze H, Jochimsen F, et al. Risk factors and outcome of 107 patients with decompensated liver disease and acute renal failure (including 26 patients with hepatorenal syndrome): the role of hemodialysis. Ren Fail. 1995; 17:135-146. [PubMed: 7644764]

45. Mitzner SR, Stange J, Klammt S, et al. Improvement of hepatorenal syndrome with extracorporeal albumin dialysis MARS: results of a prospective, randomized, controlled clinical trial. Liver Transpl. 2000; 6:277-286. [PubMed: 10827226]

46. Wong F, Raina N, Richardson R. Molecular adsorbent recirculating system is ineffective in the management of type 1 hepatorenal syndrome in patients with cirrhosis with ascites who have failed vasoconstrictor treatment. Gut. 2010; 59:381-386. [PubMed: 19710033]

47. Guevara M, Ginès P, Bandi JC, et al. Transjugular intrahepatic portosystemic shunt in hepatorenal syndrome: effects on renal function and vasoactive systems. Hepatology. 1998; 28:416-422. [PubMed: 9696006]

48. Brensing KA, Textor J, Perz J, et al. Long term outcome after transjugular intrahepatic portosystemic stent-shunt in nontransplant cirrhotics with hepatorenal syndrome: a phase II study. Gut. 2000; 47:288-295. [PubMed: 10896924]

49 -. Wong F, Leung W, Al Beshir M, et al. The outcome of patients with cirrhosis and Type-1 hepatorenal sydrome treated with liver transplantation. Liver Transpl. 2014 [Epub ahead of print]. This retrospective study of 62 patients with type 1 HRS showed that postliver transplant HRS resolved in $76 \%$ of the patients in a mean time of 13 days. The only predictor of HRS nonreversal was duration of dialysis in the pretransplant period with a $6 \%$ increase in risk of nonreversal with each additional day of dialysis.

50. Nadim MK, Sung RS, Davis CL, et al. Simultaneous liver-kidney transplantation summit: current state and future directions. Am J Transplant. 2012; 12:2901-2908. [PubMed: 22822723] 


\section{KEY POINTS}

- Diagnosis and identification of the cause of renal dysfunction in patients with cirrhosis remains challenging, particularly due to limitations associated with the use of serum creatinine to estimate GFR.

- The MDRD-6 equation seems to be superior to other standard equations at identifying patients with cirrhosis with true GFR below $30 \mathrm{ml} / \mathrm{min} / 1.73 \mathrm{~m}^{2}$. Cystatin $\mathrm{C}$ equations and cystatin C-creatinine equations performed better than typical serum creatinine equations in calculating GFR; however, their performance was lower in patients with cirrhosis than in patients without liver disease.

- NGAL and other new biomarkers have been shown to be helpful in differentiating HRS from structural kidney damage, although their use is limited to investigational purposes.

- Treatment with vasoconstrictor agents is considered to be the most effective therapy for patients with HRS type 1.

- Evaluation for liver transplantation is recommended in patients with HRS type 1 who are potential transplant candidates. 


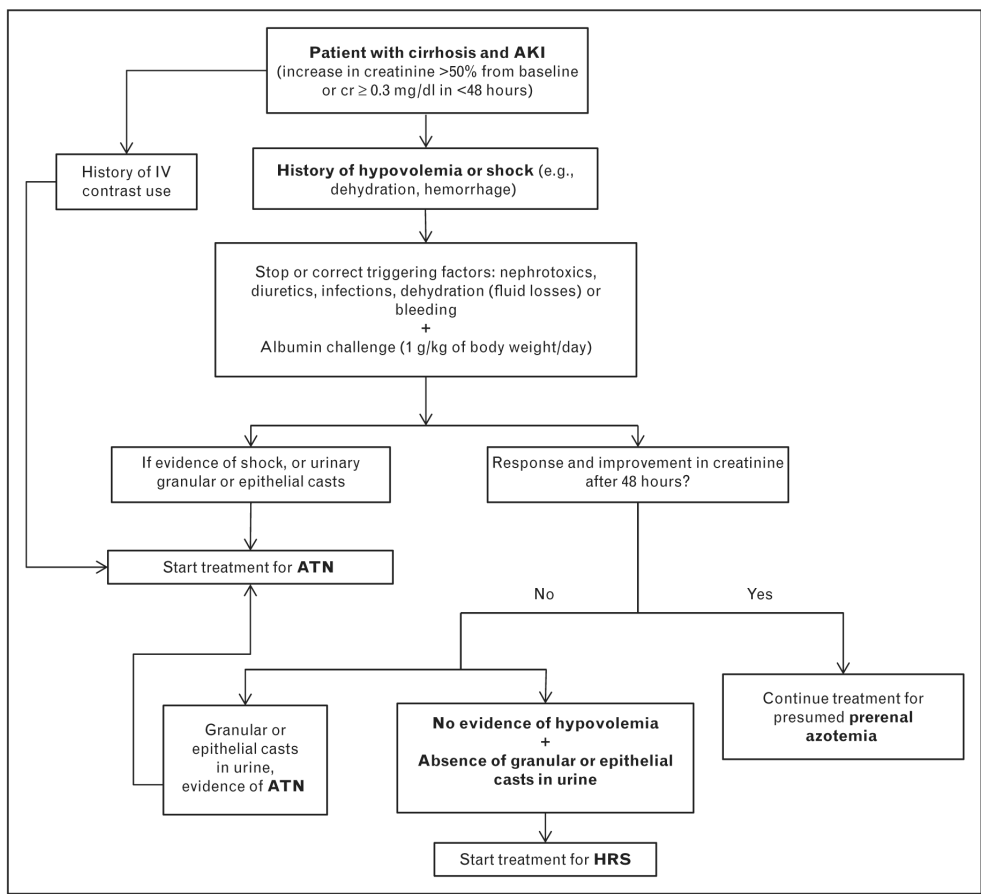

FIGURE 1.

Proposed algorithm for evaluation and management of the patient with cirrhosis and AKI. AKI, acute kidney injury; ATN, acute tubular necrosis; cr, creatinine; HRS, hepatorenal syndrome. 